\title{
Atomic Ordering in Self-assembled Epitaxial and Endotaxial Compound and Element Semiconductor Quantum Dot Structures: The First Review
}

\author{
Peter Möck \\ Portland State University, Department of Physics, P.O. Box 751, Portland, OR 97207-0751, pmoeck@pdx.edu
}

\begin{abstract}
Although the main international research thrust on self-assembled epitaxial semiconductor quantum dots is currently being directed towards random alloy quantum dots, the suggestion is made that atomically ordered quantum dots which are grown by either epitaxy or endotaxy* may in addition to their larger quantum confinement potentials possess superior long term structural stability. Such atomically ordered quantum dots should, therefore, be superior to random alloy quantum dots as far as prospective device applications are concerned. The basis for this suggestion is simple thermodynamic considerations. These considerations seem to explain our transmission electron microscopical observations of epitaxially grown atomically ordered $\mathrm{In}(\mathrm{Sb}, \mathrm{As}),(\mathrm{In}, \mathrm{Ga}) \mathrm{Sb},(\mathrm{Cd}, \mathrm{Zn}) \mathrm{Se},(\mathrm{Cd}, \mathrm{Mn}, \mathrm{Zn}) \mathrm{Se}$ quantum dots and $\mathrm{Pb}(\mathrm{Se}, \mathrm{Te})$ quantum dot predecessor islands. Atomic ordering in ( $\mathrm{In}, \mathrm{Ga}) \mathrm{P}$ quantum dot structures, as recently observed by other authors, does not seem to contradict our thermodynamic considerations. Endotaxially grown atomically ordered $(\mathrm{In}, \mathrm{Si}, \mathrm{As})$ and $(\mathrm{Sn}, \mathrm{Si})$ quantum dots in $\mathrm{Si}$ matrices are briefly discussed as an even more unconventional approach to nanostructures with applications in electronics, photonics, information storage, and sensing.
\end{abstract}

\section{INTRODUCTION}

Self-assembled epitaxial semiconductor quantum dots (QDs) were discovered in 1985 [1]. The finding in the early 90s that such QDs can possess narrow size distributions [2] led to a large international research effort that has recently been reviewed [3]. Regardless of the epitaxial deposition parameters and materials systems, alloying of the compounds or elements that constitute the QD systems is known to take place during the growth of the QDs as this is crucial for the Stranski-Krastanow growth mode to operate in semiconductors [4]. Since the epitaxial deposition and alloying are typically random events, these QD possess the structural prototype of the constituents of the alloy, i.e. the atoms are (initially) randomly distributed over their respective sublattices in the sphalerite, diamond, or halite structural prototype.

The tacit assumption throughout the vast majority of the international "quantum dot community" is that StranskiKrastanow grown random alloy QDs are structurally stable. Recent experimental observations, however, seem to indicate that this is at least for several III-V, II-VI, and IV-VI compound semiconductor systems not the case [5-12]. In these systems it appears that it is atomically ordered or phase separated QDs that are structurally stable, i.e. the initial random alloy QDs transform into ordered crystallographic or chemical superlattices. Since these transformations lead to both a smaller band gap [13] and an enhanced structural stability [7], such ordering should lead to QDs with superior properties as far as device applications are concerned.

There is already a very large body of experimental and theoretical literature which describes such transitions in heteroepitaxial semiconductor random alloy layers as consequences of both kinetics and thermodynamics [13]. The general observation is that only parts of epitaxial layers transform into atomically ordered structures. The situation with QDs is in so far different that they typically comprise only some $10^{4}$ to $10^{5}$ atoms. Their more or less complete transformation into atomically ordered structures may, therefore, be a possibility.

Well fitting to the proceedings of a Symposium on "Unconventional Approaches to Nanostructures with Applications in Electronics, Photonics, Information Storage, and Sensing", we present here the first review on atomically ordered self-assembled semiconductor QDs that were grown by epitaxy [5-12] and endotaxy [12,14-19]. On the basis of simple thermodynamics, the suggestion is made that atomically ordered quantum dots which are grown by either epitaxy or endotaxy may possess superior long term structural stability.

* The crystallographically oriented growth of a precipitate in a matrix is traditionally described as endotaxy (from the Greek, literally meaning arrangement within). 
Atomic ordering transitions in epitaxially grown (In, Ga)P, (In, Ga)Sb, In(Sb,As), (Cd,Mn, Zn)Se, (Cd,Zn)Se QDs and $\mathrm{Pb}(\mathrm{Se}, \mathrm{Te}) \mathrm{QD}$ predecessor islands [5-12] are dealt with in the first part of the paper. In the second part of the paper, we deal with endotaxially grown systems [12,14-19]. Atomic ordering in endotaxially grown (In,Si,As) and ( $\mathrm{Sn}, \mathrm{Si})$ QDs in $\mathrm{Si}$ matrices will be discussed briefly since we recently identified a void mediated formation mechanism [12,14-16] that may be employable to the endotaxial growth of atomically ordered QDs of other materials in Si matrices.

\section{THERMODYNAMIC CONSIDERATIONS AND EPITAXIALLY GROWN QUANTUM DOTS}

In thermodynamic terms, any structural transition in a QD can be explained by a reduction of its Gibbs free energy $\mathrm{G}=\mathrm{E}-\mathrm{T} \mathrm{S}+\mathrm{p} \mathrm{V}$

where $\mathrm{E}$ is the internal energy, $\mathrm{T}$ the absolute temperature, $\mathrm{S}$ the entropy, $\mathrm{p}$ the pressure, and $\mathrm{V}$ the volume of the QD. The requirement of a smaller band gap in the QD than in the surrounding matrix is usually obtained for solid solutions with larger lattice constants. This means that Stranski-Krastanow grown QDs are typically compressively strained to a few percent and we will refer to them below as ordinarily strained QDs (OS-QDs) and use the subscript os. Assuming that OS-QDs are completely embedded in a matrix with a smaller lattice constant but the same structural prototype, the product of the hydrostatic pressure on them (due to external lattice mismatch stresses) and their volume can be approximated by the product of their bulk modulus (B) and the change in their volume (dV),

$$
\mathrm{p} \mathrm{V}=\mathrm{B}_{\mathrm{os}} \mathrm{dV}_{\mathrm{os}}
$$

Now, we consider a transition from an OS-QD with the sphalerite structure into an atomically ordered QD (AOQD, subscript ${ }_{\text {ao }}$ ) with a different structural prototype and a crystallographic orientation relationship with the matrix that ensures that the atomically ordered $\mathrm{QD}$ is negligibly strained, i.e. $\mathrm{dV}_{\mathrm{ao}} \sim 0$. Combining the energy minimization principle, (1), and (2) we obtain for this kind of structural transition

$$
E_{o s}-T S_{o s}+B_{o s} d V_{o s}>E_{a o}-T S_{a o}
$$

under the condition that the contributions of the interface energies to the Gibbs free energies of the atomically ordered and random alloy QD are equal**. Solving for the critical temperature, $\mathrm{T}_{\mathrm{c}}$, of the disorder-order transition where both phases are in equilibrium, we obtain

$$
T_{c}=\frac{E_{o s}-E_{a o}+B_{o s} \cdot d V_{o s}}{S_{o s}-S_{a o}}
$$

Obviously, the larger the product $B_{o s} d V_{o s}$ in relation (4), (i.e. the effect of the external lattice mismatch stress on the OS-QDs), is, the larger $\mathrm{T}_{\mathrm{c}}$ will be.

To demonstrate the structural instability of Stranski-Krastanow grown OS-QDs, we estimated the values of the terms in (3) and (4) for one particular example, i.e. an $\mathrm{In}_{0.75} \mathrm{Ga}_{0.25} \mathrm{P} \mathrm{QD}$ embedded in a $\mathrm{Ga}_{0.75} \mathrm{In}_{0.25} \mathrm{P}$ matrix [7]. For the $3.6 \%$ lattice mismatch strain evenly distributed between the QD and the matrix (i.e. a lower bound estimate), relation (3) gave for this model system and the famatinite structural prototype of the AO-QD at $\mathrm{T}=300 \mathrm{~K}$ the structural stability determining inequality

$$
-0.66^{\mathrm{eV} / \text { atom }}+0.6^{\mathrm{eV} / \text { atom }}>-0.33^{\mathrm{eV} / \text { atom }} \text {. }
$$

This inequality means that without the external lattice mismatch stress effect (estimated to be $0.6 \mathrm{eV} /$ atom, i.e. 23 times the thermal energy of a harmonic oscillator at this temperature) on the random alloy OS-QD, it would be stable at room temperature in the sphalerite structure (as this would result in an energy reduction of $0.66{ }^{\mathrm{eV}} /$ atom). With that effect, however, the energy balance is reversed and the essentially unstrained atomically ordered (famatinite) crystallographic superlattice is the preferred structure of the QD. Due to the large elastic lattice mismatch energy contribution to the Gibbs free energy of the random alloy QD, many different atomically ordered phases may originate from such OS-QDs.

As has been shown earlier, this prediction of our thermodynamic model is borne out in experiments in a variety of binary and ternary II-VI and III-V OS-QDs and in IV-VI OS-QD predecessor island structures [7-12]. The condition of negligible external lattice mismatch stress on the AO-QDs is thereby realized by special elastic lattice mismatch energy minimizing orientation relationships, e.g. Fig. 1a.

\footnotetext{
** The contributions of the products of the atmospheric pressure and V to the Gibbs free energies are neglected.
} 
Using the strain sharing - lower bound - assumption above, we obtained from (4) as an estimate of the critical temperature

$$
T_{c}=\left(\frac{9.3234}{0.28 \cdot \ln T_{c}+T_{c} \cdot 10^{-4}}+\frac{8.5678 \cdot 10^{2}}{0.28 \cdot \ln T_{c}+T_{c} \cdot 10^{-4}}\right) K
$$

for the reversible transformation between the two possible model structures of an $\operatorname{In}_{0.75} \mathrm{Ga}_{0.25} \mathrm{P}$ QD a value of $\sim 486$ $\mathrm{K}$ [7]. The second term in this relation is due to the effect of the external lattice mismatch stress and obviously enhances $T_{c}$ significantly. Under the upper bound assumptions that all of the lattice mismatch strain is accommodated by the OS-QDs alone and the lattice of the matrix remains undistorted, we obtained $\mathrm{T}_{\mathrm{c}} \sim 852 \mathrm{~K}$, i.e. a temperature within the range that is commonly used to growth (In,Ga)P QDs [7]. This implies that atomic ordering in $(\mathrm{In}, \mathrm{Ga}) \mathrm{P}$ and similar material during epitaxial growth may be thermodynamically possible, although it is frequently discussed as a kinetic effect [13]. Such atomically ordered (In, Ga)P QDs have indeed been observed at the University of Lund $[5,6]$.

The Gibbs-Helmholtz equation (1) and the energy minimization principle tell us only which structural transformations will eventually happen, but not how fast such transitions will proceed and how high the obtainable degree of order will be. Such information can be derived qualitatively for an enhanced $T_{c}$ from the classical theories of long- [20] and short-range [21] atomic ordering as cooperative (alternatively called critical) phenomena. These theories state that at any $\mathrm{T}$ below $\mathrm{T}_{\mathrm{c}}$ the equilibrium degree of order will be the larger the higher $\mathrm{T}_{\mathrm{c}}$ is. Although the order parameter for long range order drops to zero at $\mathrm{T}_{\mathrm{c}}$ [20], short range order exists above the critical temperature to an appreciable amount [21]. Following our thermodynamic arguments, if an atomically ordered superlattice does indeed possesses the lower Gibbs free energy but $T_{c}$ is below the growth temperature, it is conceivable that over a long enough time (possibly even at room temperature [22]) long range order may develop in OS-QDs from the seeds that are provided by the short range order.

Quenched-in vacancies should play an important role in the atomic rearrangement processes in OS-QDs. In strain gradients that exist in the surroundings of OS-QDs, vacancies will move preferentially to places of higher compressive strain [23]. Provided that the kinetic energy of the vacancies is large enough to pass the tensile strain barrier in the matrix that surrounds the OS-QDs, they will end up "inside" these entities and facilitate local atomic rearrangements there.

The quotient of the number of vacancies $\left(\mathrm{n}_{\mathrm{V}}\right)$ to the number of atoms $(\mathrm{N})$ in a crystalline structure is given by

$$
\frac{n_{V}}{N}=\exp \frac{-F_{V}}{k \cdot T}
$$

where $\mathrm{F}_{\mathrm{V}}$ is the formation energy of a vacancy and $\mathrm{k}$ is Boltzmann's constant. Under realistic assumptions on the vacancy formation energy in our semiconductor systems, the effect of a fast quench from a growth temperature of $500{ }^{\circ} \mathrm{C}$ to room temperature may be an up to ten orders of magnitude higher vacancy concentration than would be in thermodynamic equilibrium [7]. Since epitaxially grown QD structures are typically free of line, area, and volume defects, ordinarily strained QDs should attract a large amount of these excess vacancies. Thermodynamics driven atomic rearrangement processes should, therefore, speed up significantly.

Without knowledge of the details of the active diffusion mechanisms, no quantitative estimates on how fast these processes are can be made. It shall, however, be noticed that compressive stress may enhance the diffusion coefficients [24] and reduce activation energies for diffusion processes [25] significantly, i.e. speed diffusion processes up.

It is, therefore, conceivable that thermodynamics driven atomic rearrangement processes may proceed even at room temperature within a few years [22]. This has been so far our explanation for transmission electron microscopical observations of atomically ordered In(Sb,As), Fig. 1a, (In, Ga)Sb, Fig. 1b, (Cd,Mn,Zn)Se, (Cd,Zn)Se QDs and $\mathrm{Pb}(\mathrm{Se}, \mathrm{Te})$ QD predecessor islands [7-12] in samples that had been stored (after the growth) at room temperature for several months up to a few years. The recently by authors from the University of Lund, Sweden, observed atomic ordering in (In, Ga)P QD structures $[5,6]$ does not contradict our thermodynamic considerations since those samples were investigated over a time scale of 2 years [26]. This may be enough time for the observed (In,Ga)P QD structures to grow from short range order that originated during the epitaxial growth. In agreement with theory [13], photoluminescence from atomically ordered (Cd,Zn)Se, (Cd,Mn,Zn)Se [8], and (In,Ga)P QDs [5,6] is observed at band gap energies that are lower than those of their random alloy counterparts.

If the atomically ordered QD are the thermodynamically more stable structures and atomic rearrangement processes are taking place over time which lead to changes in the band gap offset between QD and matrix, opto- and microelectronic devices that are designed on the basis of the random alloy band gap are expected to degrade in 
performance over time and eventually fail. As speculated earlier [7], atomic rearrangement processes may be the cause for QD lasers to fail after an operation time of about 1200 hours [27].

Although in principle structural transformations in random alloy ordinarily strained QDs seem to be feasible (and our model does indeed provide one possible explanation for a range of experimental observations [5-12]), we are aware that our simple thermodynamic arguments by no means "indicate" that producing atomically ordered quantum dots can easily be achieved. Our current strategy for demonstrating the creation of thermodynamically more stable atomically ordered QD structures is to take advantage of the significantly enhanced critical temperature, $\mathrm{T}_{\mathrm{c}}$, of OSQDs. In order to approach the equilibrium degree of order at any $\mathrm{T}$ below $\mathrm{T}_{\mathrm{c}}$, thermal treatments can be performed at this particular temperature. Since $T_{c}$ is enhanced, a relative high annealing temperature can be chosen and the approach to equilibrium will be comparatively fast. On the other hand, the annealing temperature should be sufficiently low so that the chemical compositions of the OS-QDs do not change in a manner that hampers the atomic rearrangement processes into AO-QDs. For example, if the annealing would preferentially lead to an outdiffusion of the atoms that caused the smaller band gap of the QD with respect to the matrix in the first place, the whole purpose of the heat treatment would be defeated. Finding the right heat treatment parameters for triggering structural transformations in OS-QDs by trial and error, thus, emerges as a research topic that can be considered as an unconventional approach to nanostructures. Previous in-situ transmission electron microscopy annealing studies on atomically ordered (In,Ga)Sb QDs [7,12] are encouraging that research along these lines will be rewarding, e.g. Fig. $1 b$.

Admittedly, our experimental data are at the moment more or less snapshots from different QD systems [7-12] rather than systematic support of our model in its entirety. This is because we have not yet established a long lasting systematic collaboration with an epitaxial growth group and we have not yet secured proper funding for pursuing our unconventional approach to nanostructures as forcefully as this project would require.
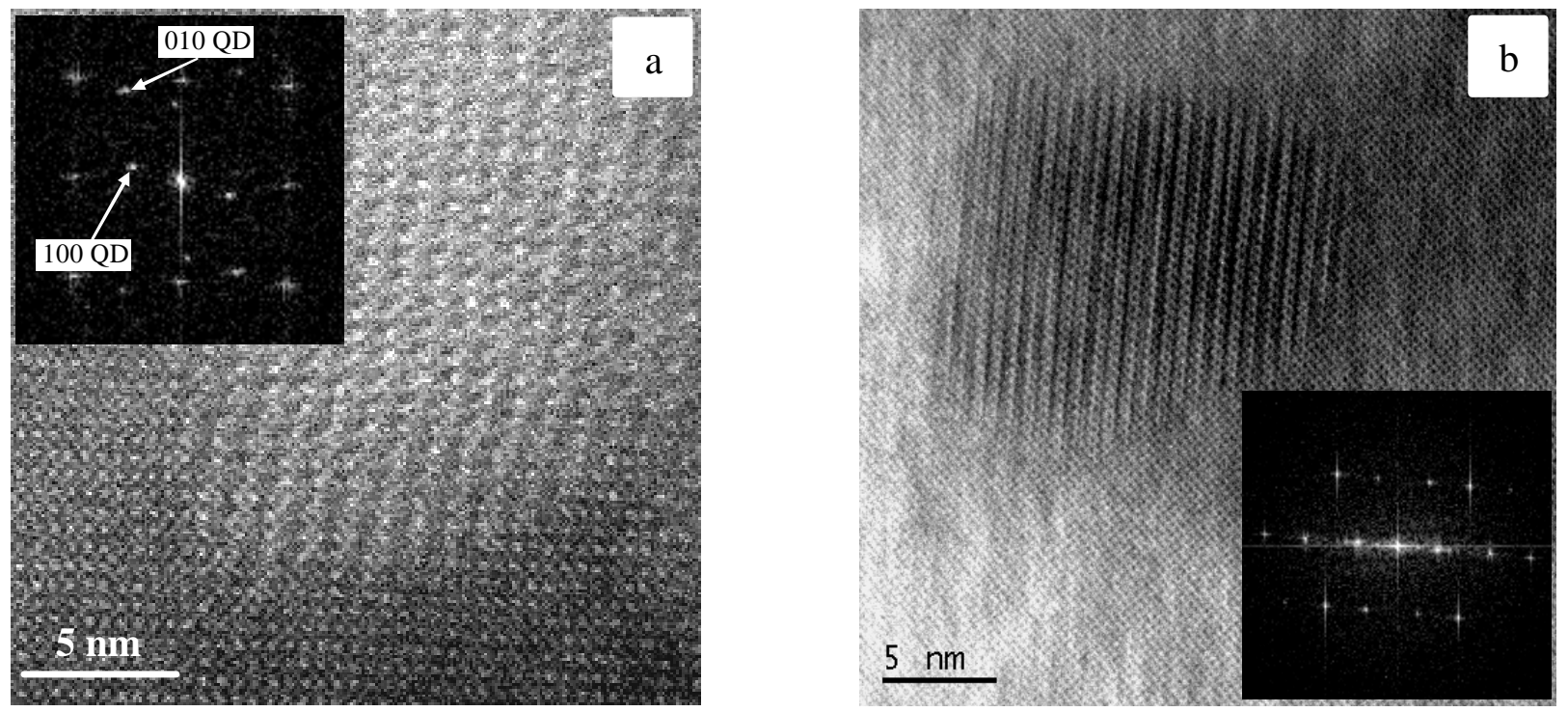

Figure 1: [001] plan views of atomically ordered quantum dots;

(a) Atomic resolution Z-contrast scanning transmission electron microscopy image of an atomically ordered In(As,Sb) QD in InAs matrix. Following the labeling in the insert Fourier transform power spectrum, the provisionally labeled (210) plane of the QD is about parallel to the (220) plane of the matrix and both reciprocal lattice vectors are of about the same magnitude. The area of the unit mesh of the atomically ordered quantum dot is in this projection about 3 times larger than that of the $\{[200],[020]\}$ unit mesh of the InAs matrix. Both of these observations are indicative of a lattice mismatch strain minimizing orientation relationship between the atomically ordered quantum dot and the matrix.

(b) High-resolution phase contrast transmission electron microscopy image of an atomically ordered (In,Ga)Sb QD in GaSb matrix. This image was recorded at $500{ }^{\circ} \mathrm{C}$ and after a several hours long heat treatment in the electron microscope at temperatures of the order of magnitude of the growth temperature. This suggests that the transformed structure of this QD rather than its initial random alloy sphalerite prototype structure is thermodynamically stable. 


\section{ENDOTAXIALLY GROWN (Sn,Si) AND (In,Si,As) QUANTUM DOTS}

As mentioned above, there are difficulties with designing the right heat treatment to facilitate atomic rearrangement processes that transform OS-QDs into thermodynamically more stable AO-QDs. Many researchers may, therefore, remain content with building devices from OS-QDs. Endotaxial growth of QDs, however, requires heat treatments as part of the growth procedure. Despite significant differences due to the specifics of the materials systems, there are also similarities in the endotaxial growth of atomically ordered (In, $\mathrm{Si}, \mathrm{As})$ and ( $\mathrm{Sn}, \mathrm{Si}) \mathrm{QDs}$ in $\mathrm{Si}$ matrices that may be of a more general nature. These similarities are low growth rates and temperatures for the heteroepitaxial growth that result in either substitutional solid $\mathrm{Sn}_{1-\mathrm{x}} \mathrm{Si}_{\mathrm{x}}$ solution quantum wells in the $\mathrm{Sn}-\mathrm{Si}$ system or in (embedded) 3D InAs QDs in the InAs-Si system. Both of these structures are then annealed at temperatures in the range of $650-900{ }^{\circ} \mathrm{C}$ for times on the order of magnitude $30 \mathrm{~min}$.

$\mathrm{Sn}_{1-\mathrm{x}} \mathrm{Si}_{\mathrm{x}}$ multi-quantum well structures that are transformed into QD multi-layer structures by heat treatments are during their epitaxial growth subjected to thermal cycling between the $\mathrm{Sn}$ deposition temperature, i.e. $140-170{ }^{\circ} \mathrm{C}$, and the Si spacer/capping layer deposition temperature, i.e. $550{ }^{\circ} \mathrm{C}[28,29]$. This repeated cooling down to the $\mathrm{Sn}$ deposition temperature may result in a large excess concentration of vacancies. In addition, low epitaxial growth temperatures typically result in large vacancy concentrations. As there is a very low line and area defect density [12], these vacancies may partly condense into equilibrium shape voids in the Si matrix. We observed recently that such voids are crucial for the operation of one of the two formation mechanisms of endotaxially grown Sn rich QDs in this material system [12,14-16].

Unrelated recent observations that voids of a similar size to our Sn rich QDs are generated about $10 \mathrm{~nm}$ below freshly prepared Si surfaces at room temperature when those get compressively stressed due to the formation of a surface oxide [30] seem to suggest that other surface stressors (such as $\mathbf{S n}_{1-\mathrm{x}} \mathrm{Si}_{\mathrm{x}}$ substitutional solution or other "quasi-sacrificial" semiconductor layers) may have the same effect at low temperatures. Voids in the Si matrix may, thus, be created. Low growth temperatures and thermal cycling may ensure that these voids reach their equilibrium shape and size. The final high temperature heat treatment may cause diffusion into these voids and eventually lead to the endotaxial formation of QDs of different systems (that might possibly even have a direct band gap) in Si matrix.

As InAs and Si possess different structures, it is not surprising that atomically ordered (In,Si,As) QDs exist in structures that are different to both the diamond and the sphalerite structure. As suspected by the authors of ref. [17] from the Max-Planck-Institute for Microstructure Physics Halle/Saale (Germany), energy minimization is probably the reason for the observed atomic rearrangements. In the case of atomically ordered ( $\mathrm{Sn}, \mathrm{Si}$ ) QDs, these entities may be stable due to nanometer size effects on the phase diagram [31,32]. Note that the bulk $\mathrm{Sn}-\mathrm{Si}$ phase diagram does not contain any $\mathrm{Sn}-\mathrm{Si}$ phases and shows at room temperature only a solid solubility of $\mathrm{Sn}$ in $\mathrm{Si}$ of $0.12 \%$.

The heat treatments that are part of the endotaxial growth procedure of QDs will ensure that these entities grow in atomic structures that are closer to the thermodynamic equilibrium than the matrix structures. As our simple thermodynamic considerations [14], the abundance of vacancies, and possible nanometer size effects on phase diagrams [31,32] suggest, one may be able to produce many different kinds of crystallographic superlattice QDs, i.e. novel nanometer sized phases. Endotaxy may, therefore, become an alternative to QD growth in the StranskiKrastanow mode since it has the inherent capacity to deliver QDs that are structurally more stable. It may, thus, also be considered as an unconventional approach to nanostructures.

\section{SUMMAY AND CONCLUSIONS}

On the basis of simple thermodynamic considerations, the suggestion is made that atomically ordered QDs which are grown by epitaxy and endotaxy may in addition to their larger quantum confinement potentials possess superior long term structural stability. Such atomically ordered QDs should, therefore, be superior to random alloy QDs as far as prospective device applications are concerned. The recently identified void mediated formation mechanism for Sn rich QDs in Si matrix may be more generally employable to the endotaxial growth of QDs of other materials in Si matrices.

\section{ACKNOWLEDGMENTS}

Much of the work quoted here was supported by a National Science Foundation grant (DMR-9733895 to Nigel D. Browning). Support from the Campus Research Board of the University of Illinois at Chicago and the Research 
Corporation is also acknowledged. I am grateful to my former colleagues: Nigel D. Browning, Yuanyuan Lei, Teya Topuria, (all University of Illinois at Chicago at that time), G. Roger Booker, Robin J. Nicholas, (both University of Oxford, U.K.), and Nigel Mason (also University of Oxford at that time) who contributed to the emerging field of atomically ordered semiconductor QDs. Harry A. Atwater (California Institute of Technology) and Regina Ragan (also California Institute of Technology at that time) are thanked for the supply of group IV QD samples and useful discussions. Jacek K. Furdyna and Malgorzata Dobrowolska (both University of Notre Dame) are thanked for the supply of II-VI QD samples. Patrick J. McCann (University of Oklahoma) is thanked for the supply of a IV-VI QD predecessor islands sample. Klaus Pierz (Physical-technical Federal Institute Braunschweig, PTB, Germany) is thanked for photoluminescence spectroscopy experiments on atomically orderd II-VI QD samples and useful discussions. Ulf Håkanson from the University of Lund (Sweden) and Nikolei D. Zakharov from the Max-PlanckInstitute for Microstructure Physics Halle/Saale (Germany) are thanked for frank discussions concerning their work on atomically ordered QDs.

\section{REFERENCES}

1. L. Goldstein et al., Appl. Phys. Lett. 47, 1099 (1985).

2. D. Leonard et al., Appl. Phys. Lett. 64, 196 (1994).

3. T.P. Pearsall (editor), Quantum Semiconductor Devices and Technologies, (Kluwer Academic Publishers, 2000).

4. A.G. Cullis et al., Phys. Rev. B 66, 81305 (2002).

5. U. Håkanson et al., Phys. Rev. B 66, 235308 (2002).

6. U. Håkanson et al., Appl. Phys. Lett. 82, 627 (2003).

7. P. Möck et al., Mater. Res. Soc. Symp. 696, N8.8 (2002).

8. P. Möck et al., Mater. Res. Soc. Symp. 749, W13.5 (2003).

9. P. Möck et al., Appl. Phys. Lett. 79, 946 (2001).

10. P. Möck et al., Mater. Res. Soc. Symp. 642, J6.3 (2001).

11. P. Möck et al., J. Electron. Mater. 30, 748 (2001).

12. P. Möck at al., Physical Chemistry of Interfaces and Nanomaterials, Jin Z. Zhang, Zhong L. Wang, Eds., Proc. of SPIE, Vol. 4807, 71 (2002).

13. A. Zunger and S. Mahajan, Atomic ordering and phase separation in epitaxial III-V alloys, in Handbook on Semiconductors (Elsevier Science B.V., 1994), Ed. T.S. Moss, Vol. 3, p. 1447.

14. P. Möck at al., Proc. 2003 Nanotechnology Conference and Trade Show, February 23 - 27, 2003, San Francisco (California), Vol. 3, pp. 74-77.

15. P. Möck at al., Mater. Res. Soc. Symp. 770, I1.7 (2003).

16. Y. Lei et al., Appl. Phys. Lett. 82, issue 24, (2003), to be published on June 16, 2003.

17. N.D. Zakharov et al., Appl. Phys. Lett. 76, 2677 (2000).

18. C.E. Cirlin et al., Mater. Sci. Engin. B 80, 108 (2001).

19. P. Werner et al., Proc. $6^{\text {th }}$ International Symposium on Advanced Physical Fields, Growth of Well-defined Nanostructures, Tsukuba, Japan, March 6 - 9, 2001, pp. 132-136.

20. W.L. Bragg and E.J. Williams, Proc. Roy. Soc. (London) A 145, 699 (1934).

21. H.A. Bethe, Proc. Roy. Soc. (London) A 150, 552 (1935).

22. The formation of (coherent) Guinier-Preston (GP1), theta" (also called GP2), and small theta' zones in Al rich $\mathrm{Al}-\mathrm{Cu}$ (and many other) alloys over time, so called "age hardening", at room temperature may serve here as an example that such processes are possible if there are quenched-in vacancies, see, e.g., R.W. Chan, "The Coming of Materials Science", (Pergamon, 2001).

23. L.A. Girifalco and D.O. Welch, "Point Defects and Diffusion in Strained Metals", (Gordon and Breach, 1967).

24. M.J. Aziz, Mater. Sci. Semicond. Process. 4, 387 (2001).

25. N.R. Zangenberg et al., Defect Diffus. Forum 194-1, 703 (2001).

26. U. Håkanson, private communication.

27. H-Y. Liu et al., Appl. Phys. Lett. 79, 2868 (2001).

28. R. Ragan et al., Mater. Sci. Engin. B 87, 204 (2001).

29. K.S. Min and H.A. Atwater, Appl. Phys. Lett. 72, 1884 (1998).

30. S.H. Lin et al., Electrochem. \& Solid State Lett. 5, G83 (2002).

31. M. Wautelet, Nanotechnology 3, 42 (1992) \& Nanotechnology 12, 68 (2001).

32. V.K. Tewary, Phys. Rev. B 66, 205321 (2002). 